\title{
Identifying global regulators in transcriptional regulatory networks in bacteria
}

\author{
Agustino Martínez-Antonio* and Julio Collado-Vides ${ }^{\dagger}$
}

The machinery for cells to take decisions, when environmental conditions change, includes protein-DNA interactions defined by transcriptional factors and their targets around promoters.

Properties of global regulators are revised attempting to reach diagnostic explicit criteria for their definition and eventual future computational identification. These include among others, the number of regulated genes, the number and type of coregulators, the different $\sigma$-classes of promoters and the number of transcriptional factors they regulate, the size of the evolutionary family they belong to, and the variety of conditions where they exert their control. As a consequence, global versus local regulation can be identified, as shown for Escherichia coli and eventually in other genomes.

\section{Addresses}

Program of Computational Genomics, CIFN, Universidad Nacional Autónoma de México A. P. 565-A Cuernavaca, Morelos, 62100, Mexico *e-mail: agustino@cifn.unam.mx

†e-mail: collado@cifn.unam.mx

\section{Current Opinion in Microbiology 2003, 6:482-489}

This review comes from a themed issue on Genomics

Edited by Eduardo A Groisman and S Dusko Ehrlich

$1369-5274 / \$$ - see front matter

(c) 2003 Elsevier Ltd. All rights reserved.

DOI 10.1016/j.mib.2003.09.002 isms, thresholds of interactions, together with the organization of genes into operons, regulons and stimulons, and the control of chromosome structure $[4,5]$.

Global regulators have been previously defined by Gottesman [6] on the basis of their pleiotropic phenotype and their ability to regulate operons that belong to different metabolic pathways. This definition excludes proteins involved in the essential cellular machinery. In this review, using what we currently know about transcriptional regulation in $E$. coli, we analyze different properties of TFs with a genomic perspective, and determine if a set of diagnostic criteria can be identified to provide an explicit way of distinguishing a global regulator from a local or dedicated regulator. The transcriptional network of $E$. coli is probably the best known for any cell, with 4405 ORFs identified [7] and an estimated $8 \%$, or roughly 300 genes as predicted or known TFs [8]. RegulonDB, a database with information on transcriptional regulation and operon organization in E. coli [9], contains experimental evidence on regulation from 105 regulators affecting 749 genes. A large number of mapped promoters and sites, as well as operons, complete the description of around $20 \%$ of the regulatory interactions of transcription in the cell. The complexity of the network of currently known interactions is shown in Figure 1.

\section{Number of genes regulated by transcription factors}

It is surprising to know that seven regulatory proteins (CRP, FNR, IHF, FIS, ArcA, NarL and Lrp) are sufficient for directly modulating the expression of $51 \%$ of genes in E. coli, see Table 1, column 2. This large influence of so few regulators is consistent with the general statistical properties of this network, the connectivity of which follows a power-law distribution [10]. In a power-law network, very few nodes have a large number of connections, whereas many nodes interact with few others in a way that the ranked distribution of number of genes $N(\mathrm{k})$ as a function of their connectivity, in a log-log plot, decays linearly with a negative exponent [11]. At the other extreme, close to one-fifth of all TFs each regulates one or two genes.

\section{Frequency of co-regulation}

As mentioned above, the expression of many genes is modulated by only a few regulators; however, the regulation by multiple TFs occurs in $49 \%$ of genes and in most cases, it seems that a global regulator works together with more specific local regulators (Table 1, column 3). Specific examples of co-regulation include, the melA $B$ 


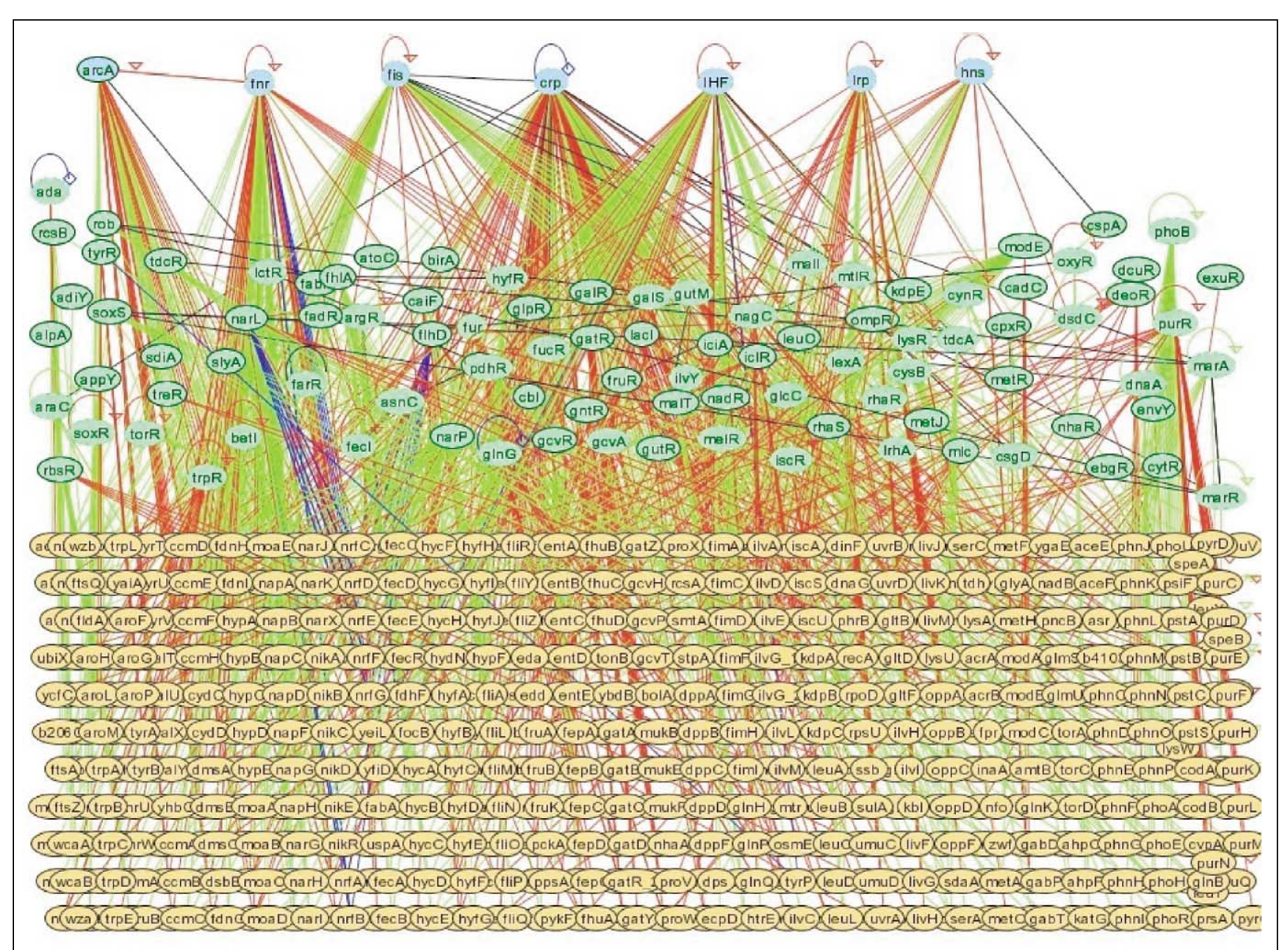

Current Opinion in Microbiology

Overview of the transcriptional regulatory network in E. coli. Regulated genes are shown as yellow ovals, TFs are shown as green ovals and TFs considered to be global regulators are shown as blue ovals. The green lines indicate activation, red lines indicate repression and dark blue lines indicate dual regulation (activation and repression).

promoter by CRP and MelR [12], ansB promoters by CRP and FNR [13], cytR regulon by CytR and CRP [14 ${ }^{\circ}$, proP2 promoter by CRP and FIS co-activation [15 ${ }^{\circ \bullet}$, met $Y$ by CRP and $\operatorname{ArgR}\left[16^{\bullet \bullet}\right.$ ], among others [17]. Of the seven TFs mentioned above, only FIS regulates most of its genes (67\%) in an independent manner. NarL and ArcA are at the other extreme as all their regulated genes are jointly co-regulated by other proteins, for instance in moe [18] and hya [19] operons. The strict co-regulation of modulons by NarL and ArcA with other TFs principally FNR and in a minor degree CRP and others, could be understood by the necessity of tight regulation between the sensing of redox potential balance between oxygen and alternative electron acceptors such as fumarate or nitrate (regulated by FNR), and the fine tuning regulation in aerobic or anaerobic responses by ArcA and NarL [20].
The mechanistic details of co-regulation vary. The active conformation of transcriptional factors before they bind to DNA in most, if not all cases, is a homo-multimeric oligomer [21]. Cooperative binding to DNA promoter operator regions increases the sensitivity for regulation $[22,23]$. For example, the same motifs of CRP involved in an interaction with the $\alpha$ subunit of RNAP are also used in its interaction with a specific TF, MelR [12]. It is not known if this is a unique accident in the recognition landscape, similar to the coincidence of the gal operator sequence and the sequence coding for the helix of the Gal regulator described years ago by Benno Müller-Hill [24]. If on the contrary, such promiscuity is a common property of specific interactions among regulators with many interactions, this would increase the possibilities for horizontal transfer of fractions of circuits and individual regulatory 
Table 1

\begin{tabular}{|c|c|c|c|c|c|c|}
\hline $\begin{array}{l}\text { Transcription } \\
\text { factor }\end{array}$ & $\begin{array}{l}\text { Genes } \\
\text { regulated* }\end{array}$ & Co-regulators $^{\dagger}$ & $\begin{array}{l}\text { TFs } \\
\text { regulated }\end{array}$ & $\begin{array}{l}\text { Sigma } \\
\text { factors }\end{array}$ & $\begin{array}{l}\text { Functional classes } \\
\text { of genes regulated }\end{array}$ & $\begin{array}{l}\text { Family } \\
\text { (members) }\end{array}$ \\
\hline CRP & 197 & 47 & 22 & $\sigma^{70,38,32,24}$ & 48 & CRP (2) \\
\hline $\mathrm{IHF}$ & 101 & 28 & 9 & $\sigma^{70,54,38}$ & 26 & HI-HNS (2) \\
\hline FNR & 111 & 20 & 5 & $\sigma^{70,54,38}$ & 22 & CRP (2) \\
\hline FIS & 76 & 15 & 4 & $\sigma^{70,38,32}$ & 20 & EBP (14) \\
\hline ArcA & 63 & 18 & 2 & $\sigma^{70,38}$ & 17 & OmpR (14) \\
\hline Lrp & 53 & 14 & 3 & $\sigma^{70,38}$ & 15 & AsnC (3) \\
\hline Hns & 26 & 14 & 5 & $\sigma^{70,38,32}$ & 17 & Histone-like (1) \\
\hline $\operatorname{NarL}^{¥}$ & 65 & 10 & 1 & $\sigma^{70,38}$ & 14 & LuxR/UhpA (17) \\
\hline OmpR & 10 & 9 & 3 & $\sigma^{70,38}$ & 5 & OmpR (14) \\
\hline Fur $\stackrel{*}{*}$ & 26 & 8 & 2 & $\sigma^{70,19}$ & 9 & Fur (2) \\
\hline PhoB & 26 & 1 & 3 & $\sigma^{70}$ & 9 & OmpR (14) \\
\hline CpxR & 9 & 2 & 1 & $\sigma^{70,38,24}$ & 5 & OmpR (14) \\
\hline SoxRS & 9 & 10 & 3 & $\sigma^{70,38}$ & 10 & AraC/XylS (24) \\
\hline $\mathrm{Mlc}^{¥}$ & 5 & 3 & 1 & $\sigma^{0,32}$ & 3 & $\mathrm{NagC/XyIR} \mathrm{(7)}$ \\
\hline $\operatorname{Csp} A^{*}$ & 2 & 2 & 1 & $\sigma^{70}$ & 2 & Cold (9) \\
\hline Rob** $^{* *}$ & 7 & 8 & 2 & $\sigma^{70,38}$ & 6 & AraC/Xyls (27) \\
\hline PurR $R^{* *}$ & 28 & 7 & 1 & $\sigma^{70}$ & 10 & GalR/Lacl (13) \\
\hline
\end{tabular}

${ }^{*}$ Total number of genes regulated directly. ${ }^{\dagger}$ Number of different TFs with which at least a gene or TU is jointly co-regulated. ${ }^{\ddagger}$ Number of regulated genes that codify for TFs. \& List of $\sigma$ factors of the regulated promoters. "Number of functional classes of the gene products regulated [44].

"TF family and in parenthesis the number of members of the family. In addition to the seven global TFs considered here there are TFs suggested by

${ }^{¥}$ Babu and Teichmann, 2003, [42"*] and **Shen-Orr et al., 2002, [50*0].

proteins, affecting in an important way the evolution of regulatory networks in bacteria [25].

\section{Regulation of transcription factors and club co-regulation}

In a process of decisions and information flux, the number of controlled or affected elements is not the only factor to be considered. A hierarchy of different levels of decision is natural to our understanding of how things get done [26]. One may consider that architecturally, the highest level in a hierarchy would be $\sigma$ factors. Certainly, $\sigma^{70}$ transcribes an enormous number of genes (830 genes compared with 31 for $\sigma^{54}$ ), as currently annotated in RegulonDB, and is therefore known for its housekeeping role. However $\sigma^{54}$, for instance, transcribes considerably less genes compared with those affected by a TF such as CRP (around 100 genes [27] compared with 200 genes). TFs amplify their range of control through the regulation of other TFs to encompass a set of indirectly regulated genes (Column 4 in Table 1). CRP is again by far, the transcription factor that regulates the most TFs (23 including itself). However, as TFs regulate other TFs, including themselves in feedback loops and complex relationships, we cannot assume a hierarchical organization of the network from these figures. Genes that are regulated by CRP also work with CRP in controlling other genes (Figure 2). In fact, 95\% of regulators that are subject to control by CRP are also 'colleagues' involved in co-regulation. Of all the 'colleagues' with which CRP works, only $45 \%$ are subject to its direct control. These ratios might be different for each regulatory protein, but in general, as observed from Table 2, global regulators work together with other global regulators (i.e. 'club co-regulation').

The dynamics of decision-making is a cooperative process of different subsets of the network put into action at certain moments. The grouping of co-regulated genes as a result of the different combinations of TFs working together in a given condition, provides the cell with the adequate flexibility to tune its transitions. This flexibility includes and could be partially analyzed in terms of the interactions and regulation among TFs.

\section{Figure 2}

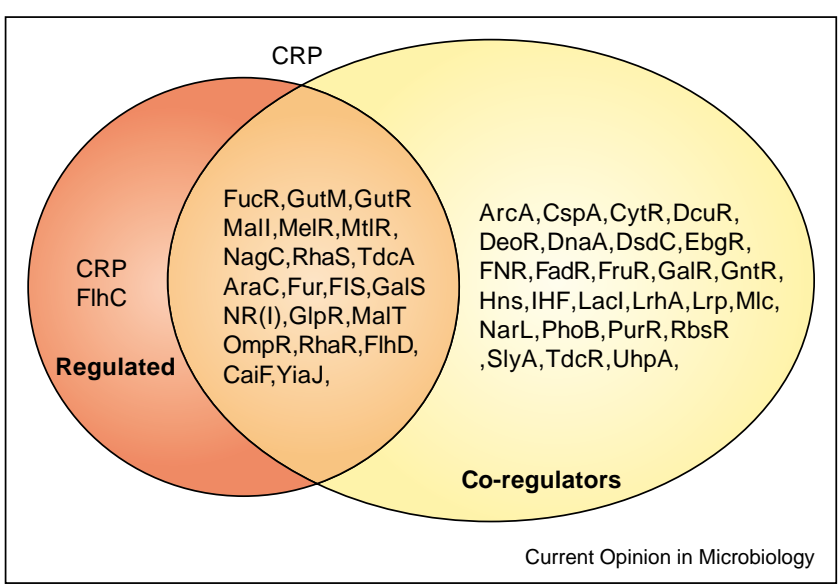

Regulated genes and co-regulators of CRP. The intersection contains those TFs that are directly regulated by CRP and which work as co-regulators with CRP. 


\begin{tabular}{ll}
\hline Table 2 & \\
\hline \multicolumn{2}{l}{ Regulatory interactions between global TFs. } \\
\hline Global regulator & $\begin{array}{l}\text { Global regulator work with } \\
\text { (co-regulators) }\end{array}$ \\
\hline CRP & IHF, FNR, ArcA, FIS, Hns, Lrp \\
IHF & CRP, FNR, ArcA, FIS, Hns, Lrp \\
FNR & CRP, IHF, ArcA, FIS, Hns, Lrp \\
Hns & CRP, IHF, FNR, ArcA, FIS \\
ArcA & CRP, IHF, FNR, FIS \\
FIS & CRP, FNR, ArcA, Hns \\
Lrp & CRP, IHF, FNR, Hns \\
\hline &
\end{tabular}

\section{Transcriptional requirements of $\sigma$ factors}

DNA-dependent RNA polymerase is the enzyme responsible for all cellular mRNA synthesis in E. coli [28]. In addition to the major housekeeping sigma factor $\sigma^{70}, E$. coli has six other $\sigma$ factors [29,30]. Changes in the synthesis of $\sigma$ factors, or competition of different $\sigma$ factors to bind to the core RNA-polymerase, even if in a delayed fashion [31], cause different programs of gene expression to be induced and repressed. In some cases this is a global mechanism for differential gene expression during cellular development $\left[32,33^{\bullet}, 34^{\bullet}\right]$, as has been shown in the very well studied differentiation of Bacillus subtilis [35,36].

A single putative global regulator is capable of regulating genes transcribed by different $\sigma$ factors (see column 5 of Table 1$)$ and $\sigma$ factors regulate genes subject to specific TFs. CRP, for instance, is able to regulate promoters transcribed by four different $\sigma$ factors. Thus the cell has more than one alternative for partitioning its genes, either by hierarchical (if they are in fact hierarchical) relationships between the different regulatory genes, or in terms of classes of genes of different $\sigma$ factor promoters. For example, a heat-shock or stationary-phase shift involves in addition to the global response by sigma factors, regulation and expression of genes involved in the precise carbon source, and nitrogen and oxygen availability. The complexity of different hierarchies and relationships shows us how the cell has encoded its actions into selected switches and groupings of genes. The cell is unlikely to have a single hierarchical perspective of regulatory networks, or a single structure-function association across species. It seems that in the same way as physiological properties of regulated genes do not depend on specific mechanisms or regulation, the decision-making distribution of tasks may be achieved differently in each organism.

TFs belonging to the $\sigma^{54}$ class are specific and rarely overlap with TFs belonging to the $\sigma^{70}$ class. Other than IHF and FNR, which have a repressor role, $\sigma^{54}$ has its own separate class of activators (e.g. NtrC, NifA and XylR), which are clearly distinct. Certainly, the mechanisms and possibilities for regulation, in particular activation, are quite different to those available for $\sigma^{70}$-like promoters [37]. The housekeeping polymerase $\sigma^{70}$ transcribes most of the genes coding for TFs, suggesting that the decision-making machinery is able to induce changes in any direction when adapting to a change in environmental conditions. In fact, only six of the 75 known regulatory genes are transcribed exclusively by an alternative $\sigma$ factor (bolA by $\sigma^{38}, \operatorname{csg} D$ by $\sigma^{38}$, htgA by $\sigma^{32}$, hycA by $\sigma^{54}$, hypF by $\sigma^{54}$ and $z r a R$ by $\sigma^{54}$ ), none of which are considered as global regulators (see below).

\section{Response to changes in environmental conditions}

The second function of transcription factors is to sense changes in environmental conditions or other internal signals encoding changes. Bacteria constantly monitor extracellular physicochemical conditions, so that they can respond by modifying their gene expression patterns to adjust growth $[38,39]$. The link between changes in environmental conditions and changes in transcriptional regulation involves signal-transduction pathways, which may involve the direct production of an isomer such as allolactose [40], or a cascade of enzymatic modifications such as the activation of the nitrogen regulator in response to nitrogen limitation [27]. The precise link with regulatory proteins is achieved by small metabolites (i.e. products of active metabolism [41]). Half of the known and predicted transcriptional regulators in E. coli have predicted domains for the binding of small metabolites, whereas $10 \%$ have a CheY-like response regulator receiver domain that are phosphorylated by kinases in two-component signal transduction systems $\left[42^{\bullet \bullet}\right]$. The role of both local and global regulators is to mediate precise activation or repression by sensing changes in specific metabolites. The only difference is that global TFs sense a larger number of growth conditions (shown in Figure 3). The concentration of cAMP must therefore be sufficient to enable CRP to perform its regulatory role [43]. As shown in column 6 of Table 1, the number of different functional classes [44] of the products whose genes are regulated by global regulators is clearly larger than the rest. Thus, not only do global regulators influence many other regulators and genes by transcriptional-specific interactions, they are also capable of sensing a larger number of environmental changes through specific metabolites.

\section{Autoregulation and isolated transcription units}

CRP is auto-regulated both positively and negatively. Lrp, FIS, IHF, Hns, FNR, Fur, PurR and SoxR among others, are negatively auto-regulated. In fact, auto-regulation is quite frequent in TFs. 55\% (58 of 105) of all TFs known are auto-regulated, and of these $68 \%$ are negatively autoregulated, $29 \%$ are positively auto-regulated (only $\mathrm{PhoB}$ of the TFs in Table 1) and 3\% are auto-regulated both positively and negatively. Thus, auto-regulation is not limited to global regulators. Negative auto-regulation, 


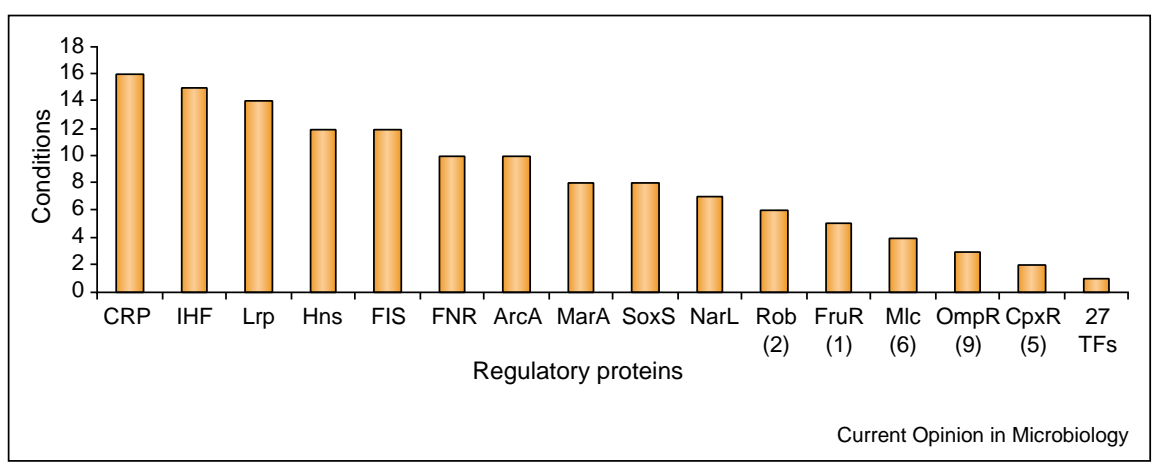

Global environment growth conditions in which TFs are regulating. To see the detailed list of conditions see RegulonDB page: http:// www.cifn.unam.mx/Computational_Genomics/regulondb/SupMat/conditions. Numbers in brackets indicate how many additional TFs participate in the same number of conditions.

which has homeostatic properties [45], is predominant with TFs with high connectivity and is also dominant in the complete network [46].

CRP, FNR, ArcA, Lrp and Hns are isolated genes with a single promoter. NarL, OmpR, CpxR and PhoB are all part of two-component systems and are coded in transcription units (TUs) with their respective sensor proteins. The gene for FIS is part of a TU with two other genes and a putative dehydrogenase with unknown function. IHF consists of two subunits each of which are in different TUs, himA as a single gene and himD in an operon with two genes and three promoters including one that expresses himD only. It is interesting to note that global TFs tend to be transcribed uncoupled from the genes they regulate, which is not usually the case for specific TFs. Indeed, $70 \%$ of global TFs are encoded in operons or TUs with at least two genes, although independent promoters could still transcribe them. Precise theoretical analyses could help to understand the dynamic benefits of such coupled and uncoupled systems $[47,48]$. The fact that global TFs are uncoupled of the genes they regulate seems an inevitable consequence of regulating many genes. However, TFs that regulate a single or very few operons encoded by a gene adjacent to the regulated operon may well be the result of horizontal gene transfer, as it would not be advantageous to acquire a new operon conferring a new function if the operon is not expressed correctly [49].

\section{Global transcription factors}

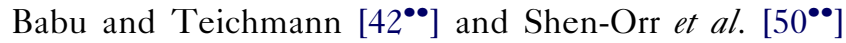
have identified global regulators in E. coli (see Table 1). Seven of them satisfy our diagnostic criteria to be considered true global regulators; CRP, IHF, FNR, FIS, ArcA, Lrp and Hns. CRP is the master regulatory protein that senses the energetic status of the cell by cAMP levels $\left[43,51^{\circ}\right]$. FNR and ArcA are directly related to energy production by regulating respiratory modes. Lrp monitors the general nutritional state by sensing L-leucine concentration in the cell and adjusts metabolism to changing nutritional conditions [52]. FIS, IHF and Hns are DNAbinding proteins that regulate gene transcription by modulating DNA topology, which is dependent on the energy levels in the cell $\left[51^{\bullet}, 52\right]$.

In a second level of hierarchy, we have identified NarL, Fur, Mlc and CspA, as also suggested by Babu and Teichmann $\left[42^{\bullet \bullet}\right.$, Rob and PurR, suggested by ShenOrr et al., [50 ${ }^{\bullet \bullet}$, as well as PhoB, CpxR and SoxR. These DNA-binding proteins are less-connected nodes, but they are notably important for cell fitness. SoxR participates in oxidative stress, and regulates SoxS, which regulates a larger number of genes than SoxR. OxyR, PdhR, ModE, FlhA, CysB, DnaA, BolA, IciA and others proteins are also likely to be positioned at this level because they start regulatory cascades, although they do not satisfy other criteria as global regulators.

Furthermore, most global regulators described here belong to evolutionary protein families, which contain a low number of members. CRP, IHF, FNR, Lrp and Hns have been classified in families with three or less members [8]. FIS, ArcA, NarL, SoxS, Cpx, OmpR and PhoB belong to families with 14 to 27 members (Table 1, column 7). These families strongly depend on the similarity of the helix-turn-helix, thus being in a family with a small number of members would in principle permit a larger variation in sequence and number of the corresponding operator sites.

Additional levels of global coordination of gene expression in addition to TFs and $\sigma$ factors include, for instance, methylation by Dam that overlaps with recognition sites for FNR and CRP [53], transient asymmetry expression of the regulatory network by chromosomal position [54], 
as well as supercoiling in association with the overall energy level of the cell $\left[31,34^{\bullet}, 51^{\bullet}, 55\right]$. In fact, transcriptional interactions defines a subset of a larger network regulation including translation, RNA antisense [56], mRNA half-life $\left[57,58^{\bullet \bullet}\right]$, transcription attenuation [59] and so forth.

\section{Concluding remarks}

This network of two-headed molecules that sense specificities of the environment, together with particular (overlapping) subsets of co-regulated genes that are expressed or repressed, describes what the cell has implemented to respond to the changes in the environment given its history in evolution. The armament for our understanding of regulatory networks, co-regulated groups, clusters and their overlapping genes, has recently increased beyond the operons, regulons and stimulons, to structurally and computationally defined motifs and modules of the network $\left[50^{\bullet \bullet}, 60^{\bullet \bullet}, 61^{\bullet \bullet}\right]$, with dynamical properties of switches, oscillators, amplitude and frequency filters $\left[62^{\bullet \bullet}\right]$, as well as with single-cell detection of transcriptional regulation and its stochasticity $\left[63^{\bullet}, 64^{\bullet \bullet}, 65,66^{\bullet \bullet}\right]$. Making biological sense in novel integrative ways seems to be closer at hand than ever before $\left[67^{\bullet \bullet}, 68\right]$. The criteria proposed here and their evaluation can be computationally implemented, and thus contribute to other methods and strategies for reconstructing regulatory networks in different genomes $\left[69^{\circ}\right]$. It is also important to evaluate whether the criteria proposed here for $E$. coli are of general validity for other organisms.

\section{Acknowledgements}

We apologize colleagues whose research was not mentioned here because of space restrictions. We acknowledge support by NIH grant number GM62205-02, and grant number 0028 from CONACYT-Mexico. We acknowledge Steve Busby for his encouragement on the writing of this review, Heladia Salgado, RM Gutiérrez and Socorro Gama for fruitful discussions and, Verónica Jiménez, Edgar Díaz and Fabiola Sánchez for their computer support.

\section{References and recommended reading}

Papers of particular interest, published within the annual period of review, have been highlighted as:

- of special interest

$\bullet$ of outstanding interest

1. Holden C: Alliance launched to model E. coli. Science 2002 , 297:1459-1460.

2. Jacob F: La Logique du Vivant, Une Histoire de L'Hérédité. Paris: Gallimard; 1970.

3. Pollack JR, Lyer VR: Characterizing the physical genome. Nat Genet 2002, 32(suppl):515-521.

4. Neidhardt FC, Savageau MA: Regulation beyond the operon. In: Cellular and Molecular Biology: Escherichia coli and Salmonella, edn 2. Edited by Neidhardt FC, Curtiss III R, Ingraham J, Lin ECC, Low KB, Magasanik B, Reznikoff W, Schaechter M, Umbarger HE and Riley M. Washington, DC: American Society for Microbiology; 1996: 1232-1245.

5. Schaechter M: Escherichia coli and Salmonella 2000: the view from here. Microbiol Mol Biol Rev 2001, 65:119-130.

6. Gottesman S: Bacterial regulation: global regulatory networks. Annu Rev Genet 1984, 18:415-441.
7. Blattner FR, Plunkett G III, Bloch CA, Perna NT, Burland V, Riley M, Collado-Vides J, Glasner JD, Rode CK, Mayhew G et al.: The complete genome sequence of Escherichia coli. Science 1997, 277:1453-1462.

8. Pérez-Rueda E, Collado-Vides J: The repertoire of DNA-binding transcriptional regulators in Escherichia coli K-12. Nucleic Acids Res 2000, 28:1838-1847.

9. Salgado H, Santos-Zavaleta A, Gama-Castro S, Millán-Zarate D, Díaz-Peredo E, Sánchez-Solano F, Pérez-Rueda E, BonavidesMartínez C, Collado-Vides J: RegulonDB (version 3.2): transcriptional regulation and operon organization in Escherichia coli K-12. Nucleic Acids Res 2001, 29:72-74. See http://www.cifn.unam.mx/Computational_Genomics/regulondb/.

10. Oosawa C, Savageau MA: Effects of alternative connectivity on behavior of randomly constructed Boolean networks. Physica $D$ 2002, 170:143-161.

11. Barabasi $A L$, Albert $R$ : Emergence of scaling in random networks. Science 1999, 286:509-512.

12. Wade JT, Belyaeva TA, Hyde El, Busby SJ: A simple mechanism for co-dependence on two activators at an Escherichia coli promoter. EMBO J 2001, 20:7160-7167.

13. Scott S, Busby S, Beacham I: Transcriptional co-activation at the ansB promoters: involvement of the activating regions of CRP and FNR when bound in tandem. Mol Microbiol 1995, 18:521-531.

14. Chahla M, Wooll J, Laue TM, Nguyen N, Senear DF: Role of

- protein-protein bridging interactions on cooperative assembly of DNA-bound CRP-CytR-CRP complex and regulation of the Escherichia coli CytR regulon. Biochemistry 2003, 42:3812-3825

The CytR operon is co-regulated by CytR and CRP. The different effects on the operon are studied here. The cytidine allosteric mediated CytR conformation makes the protein less tolerant to variation in the geometric arrangement of operator sites at different promoters.

15. McLeod SM, Aiyar SE, Gourse RL, Johnson RC: The C-terminal - domains of the RNA polymerase alpha subunits: contact site with Fis and localization during co-activation with CRP at the Escherichia coli proP P2 promoter. J Mol Biol 2002, 316:517-529. Detailed experiments are presented that explain the precise activator sites of FIS and CRP, which interact with the $\alpha$-subunits of RNA polymerase at the proP P2 promoter. Related interesting papers are Wade et al. 2001 [12] and Scott et al. 1995 [13]

16. Krin E, Laurent-Winter C, Bertin PN, Danchin A, Kolb A:

-. Transcription regulation coupling of the divergent argG and metY promoters in Escherichia coli K-12. J Bacteriol 2003, 185:3139-3146.

A paper showing that CRP can activate and repress two divergent promoters from the same site. CRP is also shown to co-regulate metY jointly with ArgR.

17. Gralla JD, Collado-Vides J: Organization and function of transcription regulatory elements. In: Cellular and Molecular Biology: Escherichia coli and Salmonella, edn 2nd. Edited by Neidhardt FC, Curtiss III R, Ingraham J, Lin ECC, Low KB, Magasanik B, Reznikoff W, Schaechter M, Umbarger HE and Riley M. Washington, DC: American Society for Microbiology; 1996, 1232-1245.

18. Hasona H, Self WT, Shanmugam KT: Transcriptional regulation of the moe (molybdate metabolism) operon of Escherichia coli. Arch Microbiol 2001, 175:178-188.

19. Richard DJ, Sawers G, Sargent F, McWalter L, Boxer DH: Transcriptional regulation in response to oxygen and nitrate of the operon encoding the [NiFe] hydrogenases 1 and 2 of Escherichia coli. Microbiol 1999, 145:2903-2912.

20. Guest JR, Green J, Irvine AS, Spiro S: The FNR modulon and FNRregulated gene expression. In: Regulation of Gene Expression in Escherichia coli. Edited by Lin ECC and Lynch AS. Texas: Chapman \& Hall; 1996, 329.

21. Wolberger C: Multiprotein-DNA complexes in transcriptional regulation. Annu Rev Biophys Biomol Struct 1999, 28:29-56.

22. Bundschuh $R$, Hayot $F$, Jayaprakash $C$ : The role of dimerization in noise reduction of simple genetic networks. J Theor Biol 2003, 220:261-269. 
23. Brent $R$, Ptashne $M$ : Mechanism of action of the lexA gene product. Proc Natl Acad Sci U S A 1981, 78:4204-4208.

24. Muller-Hill $B$, Kolkhof $P$ : DNA recognition and the code. Nature 1994 , 369:614.

25. Jain R, Rivera MC, Lake JA: Horizontal gene transfer among genomes: the complexity hypothesis. Proc Natl Acad Sci U S A 1999, 96:3801-3806.

26. Senge P: The Fifth Discipline. The Art and Practice of the Learning Organisation. London: Century; 1990.

27. Reitzer L: Nitrogen assimilation and global regulation in Escherichia coli. Annu Rev Microbiol 2003, 57:155-176.

28. Paget MS, Helmann JD: The sigma70 family of sigma factors. Genome Biol 2003, 4:203.

29. Mittenhuber G: An inventory of genes encoding RNA polymerase sigma factors in 31 completely sequenced eubacterial genomes. J Mol Microbiol Biotechnol 2002, 4:77-91.

30. Ishihama A: Functional modulation of Escherichia coli RNA polymerase. Annu Rev Microbiol 2000, 54:499-518.

31. Bordes P, Conter A, Morales V, Bouvier J, Kolb A, Gutierrez C: DNA supercoiling contributes to disconnect sigmaS accumulation from sigmaS-dependent transcription in Escherichia coli. Mol Microbiol 2003, 48:561-571.

32. Hengge-Aronis R: Signal transduction and regulatory mechanisms involved in control of the sigma(S) (RpoS) subunit of RNA polymerase. Microbiol Mol Biol Rev 2002, 66:373-395.

33. Hsu LM: Promoter clearance and escape in prokaryotes.

- Biochim Biophys Acta 2002, 1577:191-207.

The transition of open complex to a stable elongation is reviewed in detail. The kinetics of abortive initiation provides another level of mechanisms for activators of transcription.

34. Notley-McRobb L, King T, Ferenci T: rpoS mutations and loss of - general stress resistance in Escherichia coli populations as a consequence of conflict between competing stress responses. $J$ Bacteriol 2002, 184:806-811.

Surprisingly, a selective advantage was obtained, at different growth rates, by nutrient-limited bacteria that lost rpoS. Hunger responsive genes were better expressed when rpoS was lost. RpoS polymorphism in $E$. coli populations is thought to balance the conflict between the hunger response and maintenance of stress.

35. Ju J, Mitchell T, Peters H III, Haldenwang WG: Sigma factor displacement from RNA polymerase during Bacillus subtilis sporulation. J Bacteriol 1999, 181:4969-4977.

36. Fujita $\mathrm{M}$ : Temporal and selective association of multiple sigma factors with RNA polymerase during sporulation in Bacillus subtilis. Genes Cells 2000, 5:79-88.

37. Bondarenko V, Liu Y, Ninfa A, Studitsky VM: Action of prokaryotic enhancer over a distance does not require continued presence of promoter-bound sigma54 subunit. Nucleic Acids Res 2002, 30:636-642.

38. Maltsev N, Marland E, Yu GX, Bhatnagar S, Lusk R: Sentra, a database of signal transduction proteins. Nucleic Acids Res 2002, 30:349-350.

39. Rui S, Tse-Dinh YC: Topoisomerase function during bacterial responses to environmental challenge. Front Biosci 2003, 8:D256-D263.

40. Huber RE, Lytton J, Fung EB: Efflux of beta-galactosidase products from Escherichia coli. J Bacteriol 1980, 141:528-533.

41. Phelps TJ, Palumbo AV, Beliaev AS: Metabolomics and microarrays for improved understanding of phenotypic characteristics controlled by both genomics and environmental constraints. Curr Opin Biotechnol 2002, 13:20-24.

42. Babu MM, Teichmann SA: Evolution of transcription factors and

- the gene regulatory network in Escherichia coli. Nucleic Acids Res 2003, 31:1234-1244.

The authors generate computational predictions of structural comparisons of the complete set of transcription factors (TFs) in E. coli (see also Pérez-Rueda and Collado-Vides [8]). Evolutionary properties are also analyzed, including the high impact of gene duplication in generating
TFs, their internal structure as two-domain proteins, and properties of the network, cascades, global regulation, similarity of regulatory regions and connectivity of the network.

43. Busby S, Kolb A: The CAP modulon. In: Regulation of Gene Expression in Escherichia coli. Edited by: Lin ECC and Lynch AS. Texas: Chapman \& Hall; 1996, 255-270.

44. Riley M: Functions of the gene products of Escherichia coli. Microbiol Rev 1993, 57:862-952.

45. Thomas R: Laws for the dynamics of regulatory networks. Int J Dev Biol 1998, 42:479-485.

46. Thieffry D, Huerta AM, Pérez-Rueda E, Collado-Vides J: From specific gene regulation to genomic networks: a global analysis of transcriptional regulation in Escherichia coli. Bioessays 1998, 20:433-440.

47. Hlavacek WS, Savageau MA: Completely uncoupled and perfectly coupled gene expression in repressible systems. J Mol Biol 1997, 266:538-558.

48. Hlavacek WS, Savageau MA: Rules for coupled expression of regulator and effector genes in inducible circuits. $J \mathrm{Mol} B i o l$ 1996, 255:121-139.

49. Lawrence J: Selfish operons: the evolutionary impact of gene clustering in prokaryotes and eukaryotes. Curr Opin Genet Dev 1999, 9:642-648.

50. Shen-Orr SS, Milo R, Mangan S, Alon U: Network motifs in the - transcriptional regulation network of Escherichia coli. Nat Genet 2002, 31:64-68.

Based on the same notion that is used in the identification of motifs that are statistically over-represented in DNA sequences, the authors identify motifs within the $E$. coli network of transcriptional regulation (see also Thieffry et al. [46]). Three motifs were found: feedforward, single input module and dense overlapping regulons.

51. Hatfield GW, Benham CJ: DNA topology-mediated control of - global gene expression in Escherichia coli. Annu Rev Genet 2002, 36:175-203.

A review based on the proposal that supercoiling serves as a global regulator sensing cellular energy levels in $E$. coli. Different detailed mechanisms (promoter structure, IHF or FIS binding or divergent transcription) of how this is achieved in biosynthetic operons are revised.

52. Landgraf JR, Wu J, Calvo JM: Effects of nutrition and growth rate on Lrp levels in Escherichia coli. J Bacteriol 1996, 178:6930-6936.

53. Oshima T, Wada C, Kawagoe Y, Ara T, Maeda M, Masuda Y, Hiraga S, Mori H: Genome-wide analysis of deoxyadenosine methyltransferase-mediated control of gene expression in Escherichia coli. Mol Microbiol 2002, 45:673-695.

54. Dworkin J: Transient genetic asymmetry and cell fate in a bacterium. Trends Genet 2003, 19:107-112.

55. Muskhelishvili G, Travers A: Transcription factor as a topological homeostat. Front Biosci 2003, 8:D279-D285.

56. Wagner EG, Altuvia S, Romby P: Antisense RNAs in bacteria and their genetic elements. Adv Genet 2002, 46:361-398.

57. Selinger DW, Saxena RM, Cheung KJ, Church GM, Rosenow C: Global RNA half-life analysis in Escherichia coli reveals positional patterns of transcript degradation. Genome Res 2003, 13:216-223.

58. Bernstein JA, Khodursky AB, Lin PH, Lin-Chao S, Cohen SN:

- Global analysis of mRNA decay and abundance in Escherichia coli at single-gene resolution using two-color fluorescent DNA microarrays. Proc Natl Acad Sci U S A 2002, 99:9697-9702.

This is the first use of microarrays to quantify the half-life of a large number of mRNAs. Half-lives were found to be globally similar in conditions where generation times differ by a factor of three. Functionally related genes were found to have similar stabilities. See also Selinger et al. (2003) [57]

59. Mironov AS, Gusarov I, Rafikov R, Lopez LE, Shatalin K, Kreneva RA, Perumov DA, Nudler E: Sensing small molecules by nascent RNA: a mechanism to control transcription in bacteria. Cell 2002, 111:747-756.

60. Ravasz E, Somera AL, Mongru DA, Oltvai ZN, Barabasi AL:

-. Hierarchical organization of modularity in metabolic networks. Science 2002, 297:1551-1555. 
Extending previous work on power-law networks, the authors propose additional distinctions such as hierarchical and modular networks on the basis of a modular repeated duplication model. Metabolic data analyzed for a large number of organisms were shown to be modular and a clustering method based on an overlapping measure proposed to identify such modules.

61. Rosenfeld N, Elowitz MB, Alon U: Negative autoregulation -• speeds the response times of transcription networks. J Mol Biol 2002, 323:785-793.

The authors employ synthetic gene circuits in $E$. coli to measure the response time to reach half maximal product concentration of non-selfregulated and of negatively autoregulated transcription units. It is experimentally shown that negative autoregulation can significantly speed up the response time, which is related to a stronger promoter in the autoregulated case.

62. Wolf DM, Arkin AP: Motifs, modules and games in bacteria.

-. Curr Opin Microbiol 2003, 6:125-134.

A motivating review dissecting different motifs in a regulatory network in terms of architectural, biochemical and dynamical properties of switches, oscillators, amplitude, frequency and noise filters, among others. Reasons for the modularity of networks and their possible identification are also presented. Finally game theory as applied to biology is reviewed.

63. Swain PS, Elowitz MB, Siggia ED: Intrinsic and extrinsic - contributions to stochasticity in gene expression. Proc Natl Acad Sci U S A 2002, 99:12795-12800.

A theoretical study of two sources of noise in gene expression of individual cells. The origins of intrinsic and extrinsic noise are theoretically distinguishable when analyzing two genes simultaneously. The results are confirmed by simulation with plausible parameters for $E$. coli.

64. Ozbudak EM, Thattai M, Kurtser I, Grossman AD,

-. van Oudenaarden A: Regulation of noise in the expression of a single gene. Nat Genet 2002, 31:69-73.
This paper describes the biochemical and phenotypic contribution to gene expression in Bacillus subtilis by varying the rates of transcription and translation. This study provides the first experimental evidence for the biochemical origin of phenotypic noise.

65. Kamme F, Erlander MG: Global gene expression analysis of single cells. Curr Opin Drug Discov Dev 2003, 6:231-236.

66. Elowitz MB, Levine AJ, Siggia ED, Swain PS: Stochastic gene .. expression in a single cell. Science 2002, 297:1183-1186. An interesting paper in which the authors analyze phenotypic variation of individual $E$. coli cells and determine the contributions of intrinsic (from gene expression) and extrinsic (from other cellular components) noise in clonal cell populations. They find increased noise in a recA mutant suggesting that the increased noise may arise from transient parts of chromosome copy number differences.

67. Covert MW, Palsson BO: Constraints-based models: regulation -. of gene expression reduces the steady-state solution space. $J$ Theor Biol 2003, 221:309-325.

An example of a theoretical method to model metabolic and regulatory interactions that can take advantage of the current flood of genomic information. See also a historical summary account in Reed and Palsson (2003) [68].

68. Reed JL, Palsson BO: Thirteen years of building constraintbased in silico models of Escherichia coli. J Bacteriol 2003 , 185:2692-2699.

69. Qin ZS, McCue LA, Thompson W, Mayerhofer L, Lawrence CE,

- Liu JS: Identification of co-regulated genes through Bayesian clustering of predicted regulatory binding sites. Nat Biotechnol 2003, 21:435-439.

One of the latest algorithms in a series of variant methods to computationally predict binding sites based on comparisons of upstream sets of orthologous genes. 